\title{
Bio-efficacy of Different Botanicals against Thrips, Scirtothrips dorsalis Hood Infesting Bt Cotton
}

\author{
S.R. Padaliya*, R.K. Thumar, G.K. Pipaliya and J.K. Bhagora \\ Department of Agricultural Entomology, B. A. College of Agriculture, \\ Anand Agricultural University, Anand - 388110 (Gujarat), India \\ *Corresponding author
}

\section{A B S T R A C T}

\section{Keywords \\ Bio-efficacy, Bt cotton, Botanicals, Thrips \\ Article Info \\ Accepted: \\ 20 June 2018 \\ Available Online: \\ 10 July 2018}

\begin{abstract}
With a view to evaluate bio-efficacy of different botanicals against thrips, Scirtothrips dorsalis in $B t$ cotton, an experiment was conducted under field condition at Anand Agricultural University, Anand during Kharif 2017-18. Among the nine botanicals evaluated against $S$. dorsalis on Bt cotton, application ofneem seed kernel extract (NSKE)5 per cent found the most effective followed byneem oil 1 per cent, neem leaf extract (NLE) 10 per cent, garlic bulb extract (GBE) 5 per cent and ginger rhizome extract (GRE) 5 per cent with seed cotton yield 2304, 2295, 2275, 2252 and $2238 \mathrm{~kg} / \mathrm{ha}$, respectively. The highest (1: 7.34) NICBR obtained with the treatment of NLE followed by NSKE (1: 6.20) and $\operatorname{GBE}(1: 4.54)$.
\end{abstract}

\section{Introduction}

Cotton, the king of fibre reside one of the momentous and important cash crop exercising profound influence on economics and social affairs of the world. As per world cotton scenario, commercial cotton is grown in 77 countries and 123 countries are involved in the cotton related activities. The area under cotton cultivation in the world is about 29.22 million hectares with annual production of 105.71 million bales. India was leading in raw cotton production in the world during 2016-17 and production was upto 35.1 million bales of $480 \mathrm{lb}$ from 10.5 million hectares with a productivity of $568 \mathrm{~kg} / \mathrm{ha}$. Gujarat,
Maharashtra and Telangana reside the major cotton growing states contributing around 70 per cent of the area and 67per cent of cotton production in India. Gujarat ranks second in area (24.00lakh ha) and first in production (95.00 lakh bales of $170 \mathrm{~kg}$ ) in the country (Anon., 2017).Almost 148 insect pests have been reported during the whole season of cotton, out of which 17 have been designated as major insect pests of cotton crop (Abbas, 2004).After introduction of Bt cotton, the population of sucking insect pests gradually increased. Among the different sucking insect pests attacking on cotton, owing to climate change thrips, $S$. dorsalis major concerned in cotton ecosystem. Four species of thrips are 
found on cotton in Vietnam, though $S$. dorsalis and Thripspalmi Karny are the most important species. Both species are highly polyphagous, occurring on many annual crops and weeds (Capinera, 2001).

\section{Materials and Methods}

Field experiment was conducted at Anand Agricultural University, Anand during kharif season 2017-18 in Randomized Block Design with ten treatments and three replications with a view to evaluate bio-efficacy of different botanicals against $S$. dorsalis infesting $B t$ cotton. Cotton cultivar RCH-II was sown, with a spacing of $120 \mathrm{~cm}$ between two rows and 60 $\mathrm{cm}$ within the rows in gross and net area of 6.0 x $4.8 \mathrm{~m}$ and $4.8 \times 2.4 \mathrm{~m}$, respectively. Treatment wise application of insecticides were given at ETL (five thrips per leaf) on the plant by using high volume sprayer with required concentration. Subsequent three sprays were given at 10 days interval.The observations on cotton thrips were recorded from five randomly selected plants per plot before spraying and 1, 3, 5, 7 and 10 days after each sprays. The number of thrips per five leaves (three upper and two middle) were observed. The data obtained were analyzed by following standard statistical technique (Steel and Torrie, 1980).

\section{Results and Discussion}

The population of thrips was homogeneous in all the treatments before spray as treatment difference was non-significant. All the evaluated botanicals were significantly superior to control up to 10 days of spray.

\section{First spray}

One day after first spray (Table 1), the lowest (5.90/leaf) population of thrips was found in plots treated with neem seed kernel extract (NSKE)5 per cent and it was at par with neem oil 1 per cent, neem leaf extract (NLE) 10 per cent, garlic bulb extract (GBE) 5 per cent and ginger rhizome extract (GRE) 5 per cent with the population of $6.00,6.05,6.16$ and 6.36 thrips per leaf, respectively. These five treatments of botanicals found significantly superior to rest of the treatments. Among the evaluated botanicals, the highest (8.92/leaf) population of $S$. dorsalis found in plots treated with aloe vera leaf extract (AVLE) 5 per cent and it was at par with ardusa leaf extract (ALE) 10 per cent (8.68/leaf), safflower oil 1 per cent (8.74/leaf) and tulsi leaf extract (TLE) 10 per cent (8.86/leaf). More or less similar trend of efficacy was observed at three days after first spray. Population of thrips was recorded minimum (3.78/leaf) in plots treated with NSKE and it was at par with neem oil (3.95/leaf), NLE (4.04/leaf), GBE (4.12/leaf) and GRE (4.38/leaf) after five days of spray. Of the evaluated botanicals, maximum (7.62/leaf) population of thrips noticed in plots treated with AVLE and it was at par with ALE (7.06/leaf), safflower oil (7.28/leaf) and TLE (7.45/leaf). More or less analogous results were observed at seven days after first spray.

Likewise, ten days after first spray, NSKE (4.74/leaf), neem oil (4.79/leaf), NLE (4.93/leaf), GBE (5.07/leaf) and GRE (5.16/leaf) were the most effective and at par with each other. The treatments of ALE (8.03/leaf), safflower oil (8.20/leaf), TLE (8.20/leaf) and AVLE (8.32/leaf) were comparatively less effective against thrips on Bt cotton.

Pooled over periods results (Table 1) of spray revealed thatNSKE (4.52/leaf), neem oil (4.61/leaf), NLE (4.74/leaf), GBE (4.84/leaf) and GRE (5.02/leaf) were found significantly superior than rest of the evaluated botanicals. The plots treated with AVLE recorded the highest (8.14/leaf) thrips population and it was at par with ALE (7.62/leaf), safflower oil (7.85/leaf) and TLE (7.97/leaf). 


\section{Second spray}

At one day after second spray (Table 2), NSKE (3.83/leaf), neem oil (3.87/leaf), NLE (4.12/leaf), GBE (4.21/leaf) and GRE (4.43/leaf)were effective in reducing the thrips population. The plots treated with AVLE recorded the maximum (8.03/leaf) thrips population and it was at par with ALE (7.45/leaf), safflower oil (7.51/leaf) and TLE (7.68/leaf).

Population of thrips, S. dorsalis was noticed the lowest (3.58/leaf) in plots treated with NSKE and it was at par with neem oil (3.66/leaf), NLE (3.66/leaf), GBE (3.78/leaf) and GRE (3.95/leaf) after three days of spray. Among the evaluated botanicals, the maximum (6.79/leaf) population of thrips observed in plots treated with AVLE and it was at par with ALE (6.31/leaf), safflower oil (6.58/leaf) and TLE (6.63/leaf). More or less similar results were observed at five days after second spray.

The NSKE, neem oil, NLE, GBE and GRE found effective against thrips on $B t$ cotton by recording incidence between 2.85 and 3.26 per leaf at seven days after second spray. Among the evaluated botanicals, the maximum (7.01/leaf) population of $S$. dorsalis recorded in plots treated with AVLE and it was at par with ALE (6.52/leaf), safflower oil (6.58/leaf) and TLE (6.74/leaf). More or less similar trend of efficacy was observed at 10 days after second spray.

Looking to the data on pooled over periods (Table 2), the lowest (3.42/leaf) population of thrips recorded in plots treated with NSKE and it was at par with neem oil (3.50/leaf), NLE (3.62/leaf), GBE (3.74/leaf) and GRE (3.83/leaf). The plots treated with AVLE recorded the highest (7.06/leaf) thrips population and it was at par with ALE (6.63/leaf), safflower oil (6.74/leaf) and TLE (6.84/leaf).

\section{Third spray}

The lowest (3.26/leaf) incidence of thrips observed in plots treated with NSKE and it was at par with neem oil (3.46/leaf), NLE (3.54/leaf), GBE (3.66/leaf) and GRE (3.70/leaf)at one day after third spray (Table 3 ). These five botanicals found significantly superior to rest of the treatments. Of the evaluated botanicals, the maximum (6.47/leaf) population of $S$. dorsalis recorded in plots treated with AVLE and it was at par with ALE (6.00/leaf), safflower oil (6.52/leaf) and TLE (6.31/leaf). More or less resembling trend of efficacy was observed at three days after third spray.

Five days after spray, NSKE and neem oil registered the lowest (2.74/leaf) thrips incidence and it was at par with NLE (2.96/leaf), GBE (3.11/leaf) and GRE (3.22/leaf). The plots treated with AVLE recorded the highest (6.31/leaf) thrips population and it was at par with ALE (5.80/leaf), safflower oil (5.90/leaf) and TLE (6.05/leaf).

Based on the number of thrips were recorded at seven days after third spray, NSKE (1.75/leaf) found the most effective followed by neem oil (1.93/leaf), NLE (2.03/leaf), GBE (2.09/leaf) and GRE (2.22/leaf). The treatments ALE (4.79/leaf), safflower oil (4.88/leaf), TLE (4.98/leaf) and AVLE (5.16/leaf) were least effective against thrips on $B t$ cotton. More or less similar results were observed at ten days after third spray.

Pooled over periods results (Table 3 ) of third spray asserted thatNSKE (2.56/leaf), neem oil (2.67/leaf), NLE (2.78/leaf), GBE (2.92/leaf) and GRE(3.22/leaf) were found significantly superior than all the evaluated botanicals. The plots treated with AVLE recorded the highest (6.00/leaf) thrips population and it was at par with ALE (5.65/leaf), safflower oil (5.75/leaf) and TLE (5.85/leaf). 
Table.1 Bio-efficacy of botanicals against $S$. dorsalis infesting $B t$ cotton after first spray

\begin{tabular}{|c|c|c|c|c|c|c|c|c|c|}
\hline \multirow{2}{*}{$\begin{array}{l}\text { Sr. } \\
\text { No. }\end{array}$} & \multirow[t]{2}{*}{ Treatments } & \multirow{2}{*}{$\begin{array}{l}\text { Conc } \\
\dot{(\%)}\end{array}$} & \multicolumn{7}{|c|}{ No. of thrips/ leaf days after spray } \\
\hline & & & $\begin{array}{l}\text { Before } \\
\text { spray }\end{array}$ & 1 & 3 & 5 & 7 & 10 & $\begin{array}{c}\text { Pooled } \\
\text { over } \\
\text { periods }\end{array}$ \\
\hline $\mathrm{T}_{1}$ & $\begin{array}{l}\text { Neem seed kernel } \\
\text { extract }\end{array}$ & 5.0 & $\begin{array}{c}2.91 \\
(7.97)\end{array}$ & $\begin{array}{l}2.53 \mathrm{a} \\
(5.90)\end{array}$ & $\begin{array}{l}2.31 \mathrm{a} \\
(4.84)\end{array}$ & $\begin{array}{l}2.07 \mathrm{a} \\
(3.78)\end{array}$ & $\begin{array}{l}2.00 \mathrm{a} \\
(3.50)\end{array}$ & $\begin{array}{l}2.29 \mathrm{a} \\
(4.74)\end{array}$ & $\begin{array}{l}2.24 \mathrm{a} \\
(4.52)\end{array}$ \\
\hline $\mathrm{T}_{2}$ & Neem oil & 1.0 & $\begin{array}{c}2.99 \\
(8.44)\end{array}$ & $\begin{array}{l}2.55 a \\
(6.00)\end{array}$ & $\begin{array}{l}2.34 \mathrm{a} \\
(4.98)\end{array}$ & $\begin{array}{l}2.11 \mathrm{a} \\
(3.95)\end{array}$ & $\begin{array}{r}2.01 \mathrm{a} \\
(3.54)\end{array}$ & $\begin{array}{l}2.30 \mathrm{a} \\
(4.79)\end{array}$ & $\begin{array}{l}2.26 a \\
(4.61)\end{array}$ \\
\hline $\mathrm{T}_{3}$ & Safflower oil & 1.0 & $\begin{array}{c}3.05 \\
(8.80)\end{array}$ & $\begin{array}{l}3.04 b c \\
(8.74)\end{array}$ & $\begin{array}{l}2.95 b \\
(8.20)\end{array}$ & $\begin{array}{l}2.79 b \\
(7.28)\end{array}$ & $\begin{array}{l}2.71 \mathrm{c} \\
(6.84)\end{array}$ & $\begin{array}{l}2.95 \mathrm{c} \\
(8.20)\end{array}$ & $\begin{array}{l}2.89 \mathrm{~b} \\
(7.85)\end{array}$ \\
\hline $\mathrm{T}_{4}$ & $\begin{array}{l}\text { Ardusa leaf } \\
\text { extract }\end{array}$ & 10.0 & $\begin{array}{c}3.10 \\
(9.11)\end{array}$ & $\begin{array}{l}3.03 \mathrm{bc} \\
(8.68)\end{array}$ & $\begin{array}{l}2.92 b \\
(8.03)\end{array}$ & $\begin{array}{l}2.75 b \\
(7.06)\end{array}$ & $\begin{array}{l}2.64 b c \\
(6.47)\end{array}$ & $\begin{array}{l}2.92 \mathrm{bc} \\
(8.03)\end{array}$ & $\begin{array}{l}2.85 \mathrm{~b} \\
(7.62)\end{array}$ \\
\hline $\mathrm{T}_{5}$ & Tulsi leaf extract & 10.0 & $\begin{array}{c}2.98 \\
(8.38)\end{array}$ & $\begin{array}{l}3.06 \mathrm{c} \\
(8.86)\end{array}$ & $\begin{array}{l}3.00 \mathrm{~b} \\
(8.50)\end{array}$ & $\begin{array}{l}2.82 \mathrm{~b} \\
(7.45)\end{array}$ & $\begin{array}{l}2.72 c \\
(6.90)\end{array}$ & $\begin{array}{l}2.95 \mathrm{c} \\
(8.20)\end{array}$ & $\begin{array}{l}2.91 \mathrm{~b} \\
(7.97)\end{array}$ \\
\hline $\mathrm{T}_{6}$ & $\begin{array}{l}\text { Aloe vera leaf } \\
\text { extract }\end{array}$ & 5.0 & $\begin{array}{c}3.21 \\
(9.80)\end{array}$ & $\begin{array}{l}3.07 \mathrm{c} \\
(8.92)\end{array}$ & $\begin{array}{l}3.02 b \\
(8.62)\end{array}$ & $\begin{array}{l}2.85 b \\
(7.62)\end{array}$ & $\begin{array}{l}2.80 \mathrm{c} \\
(7.34)\end{array}$ & $\begin{array}{l}2.97 \mathrm{c} \\
(8.32)\end{array}$ & $\begin{array}{l}2.94 b \\
(8.14)\end{array}$ \\
\hline $\mathrm{T}_{7}$ & Neem leaf extract & 10.0 & $\begin{array}{c}2.70 \\
(6.79)\end{array}$ & $\begin{array}{l}2.56 a \\
(6.05)\end{array}$ & $\begin{array}{l}2.36 \mathrm{a} \\
(5.07)\end{array}$ & $\begin{array}{l}2.13 \mathrm{a} \\
(4.04)\end{array}$ & $\begin{array}{r}2.07 \mathrm{a} \\
(3.78)\end{array}$ & $\begin{array}{l}2.33 \mathrm{ab} \\
(4.93)\end{array}$ & $\begin{array}{l}2.29 a \\
(4.74)\end{array}$ \\
\hline $\mathrm{T}_{8}$ & $\begin{array}{ll}\text { Garlic } & \text { bulb } \\
\text { extract } & \end{array}$ & 5.0 & $\begin{array}{c}3.33 \\
(10.59)\end{array}$ & $\begin{array}{l}2.58 \mathrm{a} \\
(6.16)\end{array}$ & $\begin{array}{l}2.37 \mathrm{a} \\
(5.12)\end{array}$ & $\begin{array}{l}2.15 \mathrm{a} \\
(4.12)\end{array}$ & $\begin{array}{c}2.08 \mathrm{ab} \\
(3.83)\end{array}$ & $\begin{array}{l}2.36 \mathrm{ab} \\
(5.07)\end{array}$ & $\begin{array}{l}2.31 \mathrm{a} \\
(4.84)\end{array}$ \\
\hline $\mathrm{T}_{9}$ & $\begin{array}{l}\text { Ginger rhizome } \\
\text { extract }\end{array}$ & 5.0 & $\begin{array}{l}2.95 \\
(8.20)\end{array}$ & $\begin{array}{c}2.62 \mathrm{ab} \\
(6.36)\end{array}$ & $\begin{array}{r}2.40 \mathrm{a} \\
(5.26)\end{array}$ & $\begin{array}{l}2.21 \mathrm{a} \\
(4.38)\end{array}$ & $\begin{array}{l}2.13 \mathrm{ab} \\
(4.04)\end{array}$ & $\begin{array}{l}2.38 \mathrm{ab} \\
(5.16)\end{array}$ & $\begin{array}{l}2.35 \mathrm{a} \\
(5.02)\end{array}$ \\
\hline $\mathrm{T}_{10}$ & $\begin{array}{l}\text { Control } \\
\text { (Water spray) }\end{array}$ & - & $\begin{array}{c}3.13 \\
(9.30)\end{array}$ & $\begin{array}{c}3.50 \mathrm{~d} \\
(11.75 \\
)\end{array}$ & $\begin{array}{c}3.53 \mathrm{c} \\
(11.96 \\
)\end{array}$ & $\begin{array}{c}3.38 \mathrm{c} \\
(10.92 \\
)\end{array}$ & $\begin{array}{c}3.45 \mathrm{~d} \\
(11.40 \\
\quad)\end{array}$ & $\begin{array}{c}3.55 d \\
(12.10)\end{array}$ & $\begin{array}{c}3.48 c \\
(11.61)\end{array}$ \\
\hline \multicolumn{3}{|c|}{$\begin{array}{l}\text { S. Em. } \pm \\
\text { T }\end{array}$} & 0.20 & 0.13 & 0.15 & 0.16 & 0.17 & 0.18 & 0.06 \\
\hline & & $\mathbf{P}$ & - & - & - & - & - & - & 0.05 \\
\hline & & $\mathbf{T} \times \mathbf{P}$ & - & - & - & - & - & - & 0.15 \\
\hline & & V. \% & 11.52 & 8.02 & 9.72 & 11.10 & 11.89 & 11.32 & 10.34 \\
\hline
\end{tabular}


Table.2 Bio-efficacy of botanicals against $S$. dorsalis infesting $B t$ cotton after second spray

\begin{tabular}{|c|c|c|c|c|c|c|c|c|}
\hline \multirow{2}{*}{$\begin{array}{l}\text { Sr. } \\
\text { No. }\end{array}$} & \multirow[t]{2}{*}{ Treatments } & \multirow{2}{*}{$\begin{array}{l}\text { Conc. } \\
(\%)\end{array}$} & \multicolumn{6}{|c|}{ No. of thrips/ leaf days after spray } \\
\hline & & & 1 & 3 & 5 & 7 & 10 & $\begin{array}{c}\text { Pooled } \\
\text { over } \\
\text { periods }\end{array}$ \\
\hline $\mathrm{T}_{1}$ & $\begin{array}{l}\text { Neem seed kernel } \\
\text { extract }\end{array}$ & 5.0 & $\begin{array}{l}2.08 \mathrm{a} \\
(3.83)\end{array}$ & $\begin{array}{l}2.02 \mathrm{a} \\
(3.58)\end{array}$ & $\begin{array}{l}1.86 \mathrm{a} \\
(2.96)\end{array}$ & $\begin{array}{l}1.83 \mathrm{a} \\
(2.85)\end{array}$ & $\begin{array}{l}2.10 \mathrm{a} \\
(3.91)\end{array}$ & $\begin{array}{l}1.98 \mathrm{a} \\
(3.42)\end{array}$ \\
\hline $\mathrm{T}_{2}$ & Neem oil & 1.0 & $\begin{array}{l}2.09 a \\
(3.87)\end{array}$ & $\begin{array}{l}2.04 a \\
(3.66)\end{array}$ & $\begin{array}{l}1.89 \mathrm{a} \\
(3.07)\end{array}$ & $\begin{array}{l}1.86 a \\
(2.96)\end{array}$ & $\begin{array}{l}2.12 \mathrm{a} \\
(3.99)\end{array}$ & $\begin{array}{l}2.00 \mathrm{a} \\
(3.50)\end{array}$ \\
\hline $\mathrm{T}_{3}$ & Safflower oil & 1.0 & $\begin{array}{l}2.83 \mathrm{~b} \\
(7.51)\end{array}$ & $\begin{array}{l}2.66 b \\
(6.58)\end{array}$ & $\begin{array}{l}2.56 \mathrm{c} \\
(6.05)\end{array}$ & $\begin{array}{l}2.66 b \\
(6.58)\end{array}$ & $\begin{array}{l}2.76 b c \\
(7.12)\end{array}$ & $\begin{array}{l}2.69 b \\
(6.74)\end{array}$ \\
\hline $\mathrm{T}_{4}$ & Ardusa leaf extract & 10.0 & $\begin{array}{l}2.82 \mathrm{~b} \\
(7.45)\end{array}$ & $\begin{array}{l}2.61 \mathrm{~b} \\
(6.31)\end{array}$ & $\begin{array}{l}2.53 b c \\
(5.90)\end{array}$ & $\begin{array}{l}2.65 b \\
(6.52)\end{array}$ & $\begin{array}{l}2.73 b c \\
(6.95)\end{array}$ & $\begin{array}{l}2.67 b \\
(6.63)\end{array}$ \\
\hline $\mathrm{T}_{5}$ & Tulsi leaf extract & 10.0 & $\begin{array}{l}2.86 b \\
(7.68)\end{array}$ & $\begin{array}{l}2.67 b \\
(6.63)\end{array}$ & $\begin{array}{l}2.56 \mathrm{c} \\
(6.05)\end{array}$ & $\begin{array}{l}2.69 b \\
(6.74)\end{array}$ & $\begin{array}{l}2.77 \mathrm{c} \\
(7.17)\end{array}$ & $\begin{array}{l}2.71 \mathrm{~b} \\
(6.84)\end{array}$ \\
\hline $\mathrm{T}_{6}$ & $\begin{array}{l}\text { Aloe vera leaf } \\
\text { extract }\end{array}$ & 5.0 & $\begin{array}{l}2.92 \mathrm{~b} \\
(8.03)\end{array}$ & $\begin{array}{l}2.70 b \\
(6.79)\end{array}$ & $\begin{array}{l}2.60 \mathrm{c} \\
(6.26)\end{array}$ & $\begin{array}{l}2.74 b \\
(7.01)\end{array}$ & $\begin{array}{l}2.79 c \\
(7.28)\end{array}$ & $\begin{array}{l}2.75 b \\
(7.06)\end{array}$ \\
\hline $\mathrm{T}_{7}$ & Neem leaf extract & 10.0 & $\begin{array}{l}2.15 \mathrm{a} \\
(4.12)\end{array}$ & $\begin{array}{l}2.04 \mathrm{a} \\
(3.66)\end{array}$ & $\begin{array}{r}1.91 \mathrm{a} \\
(3.15)\end{array}$ & $\begin{array}{r}1.88 \mathrm{a} \\
(3.03)\end{array}$ & $\begin{array}{l}2.16 \mathrm{a} \\
(4.17)\end{array}$ & $\begin{array}{l}2.03 \mathrm{a} \\
(3.62)\end{array}$ \\
\hline $\mathrm{T}_{8}$ & Garlic bulb extract & 5.0 & $\begin{array}{l}2.17 \mathrm{a} \\
(4.21)\end{array}$ & $\begin{array}{l}2.07 \mathrm{a} \\
(3.78)\end{array}$ & $\begin{array}{l}1.93 \mathrm{a} \\
(3.22)\end{array}$ & $\begin{array}{l}1.92 \mathrm{a} \\
(3.19)\end{array}$ & $\begin{array}{l}2.19 \mathrm{ab} \\
(4.30)\end{array}$ & $\begin{array}{l}2.06 \mathrm{a} \\
(3.74)\end{array}$ \\
\hline $\mathrm{T}_{9}$ & $\begin{array}{l}\text { Ginger rhizome } \\
\text { extract }\end{array}$ & 5.0 & $\begin{array}{l}2.22 \mathrm{a} \\
(4.43)\end{array}$ & $\begin{array}{l}2.11 \mathrm{a} \\
(3.95)\end{array}$ & $\begin{array}{l}1.95 \mathrm{ab} \\
(3.30)\end{array}$ & $\begin{array}{r}1.94 \mathrm{a} \\
(3.26)\end{array}$ & $\begin{array}{c}2.19 \mathrm{ab} \\
(4.30)\end{array}$ & $\begin{array}{l}2.08 \mathrm{a} \\
(3.83)\end{array}$ \\
\hline $\mathrm{T}_{10}$ & $\begin{array}{l}\text { Control (Water } \\
\text { spray) }\end{array}$ & - & $\begin{array}{c}3.63 \mathrm{c} \\
(12.67)\end{array}$ & $\begin{array}{c}3.54 \mathrm{c} \\
(12.03)\end{array}$ & $\begin{array}{c}3.53 \mathrm{~d} \\
(11.96)\end{array}$ & $\begin{array}{c}3.41 \mathrm{c} \\
(11.13)\end{array}$ & $\begin{array}{c}3.50 \mathrm{~d} \\
(11.75)\end{array}$ & $\begin{array}{c}3.52 \mathrm{c} \\
(11.89)\end{array}$ \\
\hline \multicolumn{2}{|c|}{ S. Em. \pm} & $\mathrm{T}$ & 0.16 & 0.15 & 0.16 & 0.16 & 0.18 & 0.06 \\
\hline & & $\mathrm{P}$ & - & - & - & - & - & 0.05 \\
\hline & & $\mathrm{T} \times \mathrm{P}$ & - & - & - & - & - & 0.16 \\
\hline & & C.V. \% & 11.08 & 10.33 & 12.11 & 11.66 & 12.10 & 11.59 \\
\hline
\end{tabular}


Table.3 Bio-efficacy of botanicals against $S$. dorsalis infesting $B t$ cotton after third spray and pooled over sprays

\begin{tabular}{|c|c|c|c|c|c|c|c|c|c|}
\hline \multirow{2}{*}{$\begin{array}{l}\text { Sr. } \\
\text { No. }\end{array}$} & \multirow{2}{*}{ Treatments } & \multirow{2}{*}{$\begin{array}{l}\text { Conc. } \\
(\%)\end{array}$} & \multicolumn{6}{|c|}{ No. of thrips/ leaf days after spray } & \multirow{2}{*}{$\begin{array}{c}\text { Pooled } \\
\text { over } \\
\text { sprays }\end{array}$} \\
\hline & & & 1 & 3 & 5 & 7 & 10 & $\begin{array}{c}\text { Pooled } \\
\text { over } \\
\text { periods }\end{array}$ & \\
\hline $\mathrm{T}_{1}$ & $\begin{array}{l}\text { Neem seed kernel } \\
\text { extract }\end{array}$ & 5.0 & $\begin{array}{r}1.94 \mathrm{a} \\
(3.26)\end{array}$ & $\begin{array}{l}1.85 a \\
(2.92)\end{array}$ & $\begin{array}{l}1.80 \mathrm{a} \\
(2.74)\end{array}$ & $\begin{array}{l}1.50 \mathrm{a} \\
(1.75)\end{array}$ & $\begin{array}{l}1.64 \mathrm{a} \\
(2.19)\end{array}$ & $\begin{array}{l}1.75 \mathrm{a} \\
(2.56)\end{array}$ & $\begin{array}{l}1.99 a \\
(3.46)\end{array}$ \\
\hline $\mathrm{T}_{2}$ & Neem oil & 1.0 & $\begin{array}{l}1.99 \mathrm{a} \\
(3.46)\end{array}$ & $\begin{array}{l}1.87 \mathrm{a} \\
(3.00)\end{array}$ & $\begin{array}{l}1.80 \mathrm{a} \\
(2.74)\end{array}$ & $\begin{array}{l}1.56 \mathrm{a} \\
(1.93)\end{array}$ & $\begin{array}{l}1.67 \mathrm{a} \\
(2.29)\end{array}$ & $\begin{array}{l}1.78 \mathrm{a} \\
(2.67)\end{array}$ & $\begin{array}{l}2.01 \mathrm{a} \\
(3.54)\end{array}$ \\
\hline $\mathrm{T}_{3}$ & Safflower oil & 1.0 & $\begin{array}{c}2.56 b c \\
(6.52)\end{array}$ & $\begin{array}{l}2.59 \mathrm{~b} \\
(6.21)\end{array}$ & $\begin{array}{l}2.53 \mathrm{~b} \\
(5.90)\end{array}$ & $\begin{array}{l}2.32 \mathrm{~b} \\
(4.88)\end{array}$ & $\begin{array}{l}2.47 \mathrm{~b} \\
(5.60)\end{array}$ & $\begin{array}{l}2.50 \mathrm{~b} \\
(5.75)\end{array}$ & $\begin{array}{l}2.69 \mathrm{~b} \\
(6.74)\end{array}$ \\
\hline $\mathrm{T}_{4}$ & Ardusa leaf extract & 10.0 & $\begin{array}{l}2.55 b c \\
(6.00)\end{array}$ & $\begin{array}{l}2.59 \mathrm{~b} \\
(6.21)\end{array}$ & $\begin{array}{l}2.51 b \\
(5.80)\end{array}$ & $\begin{array}{l}2.30 \mathrm{~b} \\
(4.79)\end{array}$ & $\begin{array}{l}2.43 b \\
(5.40)\end{array}$ & $\begin{array}{l}2.48 b \\
(5.65)\end{array}$ & $\begin{array}{l}2.66 \mathrm{~b} \\
(6.58)\end{array}$ \\
\hline $\mathrm{T}_{5}$ & Tulsi leaf extract & 10.0 & $\begin{array}{l}2.61 \mathrm{c} \\
(6.31)\end{array}$ & $\begin{array}{l}2.60 \mathrm{~b} \\
(6.26)\end{array}$ & $\begin{array}{l}2.56 b \\
(6.05)\end{array}$ & $\begin{array}{l}2.34 b \\
(4.98)\end{array}$ & $\begin{array}{l}2.49 \mathrm{~b} \\
(5.70)\end{array}$ & $\begin{array}{l}2.52 b \\
(5.85)\end{array}$ & $\begin{array}{l}2.71 b \\
(6.84)\end{array}$ \\
\hline $\mathrm{T}_{6}$ & $\begin{array}{l}\text { Aloe vera leaf } \\
\text { extract }\end{array}$ & 5.0 & $\begin{array}{l}2.64 c \\
(6.47)\end{array}$ & $\begin{array}{l}2.64 b \\
(6.47)\end{array}$ & $\begin{array}{l}2.61 b \\
(6.31)\end{array}$ & $\begin{array}{l}2.38 b \\
(5.16)\end{array}$ & $\begin{array}{l}2.50 \mathrm{~b} \\
(5.75)\end{array}$ & $\begin{array}{l}2.55 b \\
(6.00)\end{array}$ & $\begin{array}{l}2.74 b \\
(7.01)\end{array}$ \\
\hline $\mathrm{T}_{7}$ & Neem leaf extract & 10.0 & $\begin{array}{l}2.01 \mathrm{a} \\
(3.54)\end{array}$ & $\begin{array}{l}1.91 \mathrm{a} \\
(3.15)\end{array}$ & $\begin{array}{l}1.86 \mathrm{a} \\
(2.96)\end{array}$ & $\begin{array}{l}1.59 \mathrm{a} \\
(2.03)\end{array}$ & $\begin{array}{l}1.69 \mathrm{a} \\
(2.36)\end{array}$ & $\begin{array}{l}1.81 \mathrm{a} \\
(2.78)\end{array}$ & $\begin{array}{l}2.04 a \\
(3.66)\end{array}$ \\
\hline $\mathrm{T}_{8}$ & Garlic bulb extract & 5.0 & $\begin{array}{c}2.04 \mathrm{ab} \\
(3.66)\end{array}$ & $\begin{array}{l}1.94 \mathrm{a} \\
(3.26)\end{array}$ & $\begin{array}{l}1.90 \mathrm{a} \\
(3.11)\end{array}$ & $\begin{array}{l}1.61 \mathrm{a} \\
(2.09)\end{array}$ & $\begin{array}{l}1.74 \mathrm{a} \\
(2.53)\end{array}$ & $\begin{array}{l}1.85 \mathrm{a} \\
(2.92)\end{array}$ & $\begin{array}{l}2.07 \mathrm{a} \\
(3.78)\end{array}$ \\
\hline $\mathrm{T}_{9}$ & $\begin{array}{l}\text { Ginger rhizome } \\
\text { extract }\end{array}$ & 5.0 & $\begin{array}{l}2.05 \mathrm{ab} \\
(3.70)\end{array}$ & $\begin{array}{r}1.97 \mathrm{a} \\
(3.38)\end{array}$ & $\begin{array}{l}1.93 \mathrm{a} \\
(3.22)\end{array}$ & $\begin{array}{l}1.65 \mathrm{a} \\
(2.22)\end{array}$ & $\begin{array}{l}1.76 a \\
(2.60)\end{array}$ & $\begin{array}{l}1.87 \mathrm{a} \\
(3.22)\end{array}$ & $\begin{array}{l}2.10 \mathrm{a} \\
(3.91)\end{array}$ \\
\hline $\mathrm{T}_{10}$ & $\begin{array}{l}\text { Control (Water } \\
\text { spray) }\end{array}$ & - & $\begin{array}{c}3.51 \mathrm{~d} \\
(11.82)\end{array}$ & $\begin{array}{c}3.49 \mathrm{c} \\
(11.68)\end{array}$ & $\begin{array}{c}3.54 \mathrm{c} \\
(12.03)\end{array}$ & $\begin{array}{c}3.44 \mathrm{c} \\
(11.33)\end{array}$ & $\begin{array}{c}3.56 \mathrm{c} \\
(12.17)\end{array}$ & $\begin{array}{c}3.51 \mathrm{c} \\
(11.82)\end{array}$ & $\begin{array}{c}3.50 \mathrm{c} \\
(11.75)\end{array}$ \\
\hline \multicolumn{2}{|c|}{ S. Em. \pm} & $\mathrm{T}$ & 0.16 & 0.16 & 0.17 & 0.15 & 0.15 & 0.07 & 0.06 \\
\hline & & $\mathrm{P}$ & - & - & - & - & - & 0.05 & 0.05 \\
\hline & & $\mathrm{T} \times \mathrm{P}$ & - & - & - & - & - & 0.15 & 0.08 \\
\hline & & C.V. \% & 11.85 & 11.48 & 12.80 & 12.77 & 11.79 & 11.60 & 11.07 \\
\hline \multicolumn{9}{|c|}{ Notes: Figures in parentheses are retransformed values of $\sqrt{x+0.5}$} & \\
\hline
\end{tabular}




\begin{tabular}{|c|c|c|c|c|}
\hline \multicolumn{5}{|c|}{ Table.4 Effect of various botanicals on seed cotton yield and economics } \\
\hline $\begin{array}{l}\text { Sr. } \\
\text { No. }\end{array}$ & Treatments & \multirow{2}{*}{$\begin{array}{c}\begin{array}{c}\text { Yield } \\
(\mathrm{kg} / \mathrm{ha})\end{array} \\
2304 \mathrm{a}\end{array}$} & \multirow{2}{*}{$\begin{array}{c}\text { Increase in yield } \\
\text { over control (\%) } \\
49.71\end{array}$} & \multirow{2}{*}{$\begin{array}{c}\text { NICBR } \\
1: 6.20\end{array}$} \\
\hline $\mathrm{T}_{1}$ & $\begin{array}{l}\text { Neem seed kernel } \\
\text { extract }\end{array}$ & & & \\
\hline $\mathrm{T}_{2}$ & Neem oil & $2295 \mathrm{a}$ & 49.12 & $1: 2.85$ \\
\hline $\mathrm{T}_{3}$ & Safflower oil & $1879 b$ & 22.09 & 1:0.80 \\
\hline $\mathrm{T}_{4}$ & Ardusa leaf extract & $1902 b$ & 23.59 & 1:-0.04 \\
\hline $\mathrm{T}_{5}$ & Tulsi leaf extract & $1860 \mathrm{~b}$ & 20.86 & 1:-0.15 \\
\hline $\mathrm{T}_{6}$ & $\begin{array}{l}\text { Aloe vera leaf } \\
\text { extract }\end{array}$ & $1845 b$ & 19.88 & $1: 2.65$ \\
\hline $\mathrm{T}_{7}$ & Neem leaf extract & $2275 \mathrm{a}$ & 47.82 & $1: 7.34$ \\
\hline $\mathrm{T}_{8}$ & Garlic bulb extract & $2252 \mathrm{a}$ & 46.33 & $1: 4.54$ \\
\hline $\mathrm{T}_{9}$ & $\begin{array}{l}\text { Ginger rhizome } \\
\text { extract }\end{array}$ & $2238 \mathrm{a}$ & 45.42 & $1: 2.43$ \\
\hline $\mathrm{T}_{10}$ & $\begin{array}{l}\text { Control (Water } \\
\text { spray) }\end{array}$ & $1539 \mathrm{c}$ & - & - \\
\hline \multicolumn{2}{|c|}{ S. Em. \pm} & 99.26 & - & - \\
\hline \multicolumn{2}{|c|}{ C. V. $(\%)$} & 8.43 & - & - \\
\hline \multicolumn{5}{|c|}{$\begin{array}{l}\text { Note: Treatment mean with letter(s) in common are non-significant by DNMRT at } 5 \% \\
\text { significance }\end{array}$} \\
\hline
\end{tabular}




\section{Fig. 1: Bio-efficacy of different botanicals against $S$. dorsalis infestingBt cotton (Pooled over sprays)}

T1: Neem seed kernel extract, $5.0 \%$ $5.0 \%$

T2: Neem oil, $1.0 \%$

T3: Safflower oil, $1.0 \%$
T5: Aloe vera leaf extract,

T6: Neem leaf extract, $10.0 \%$

T7: Garlic bulb extract $5.0 \%$

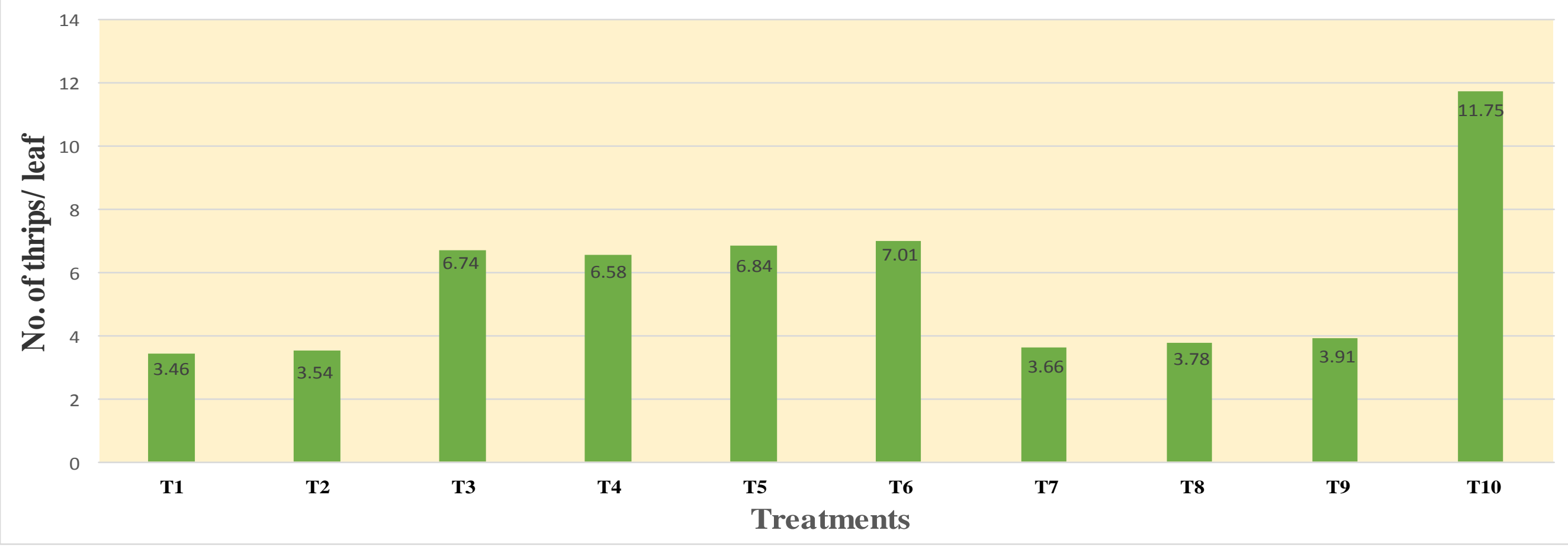




\section{Fig. 2: Effect of different botanical treatments on seed cotton yield}

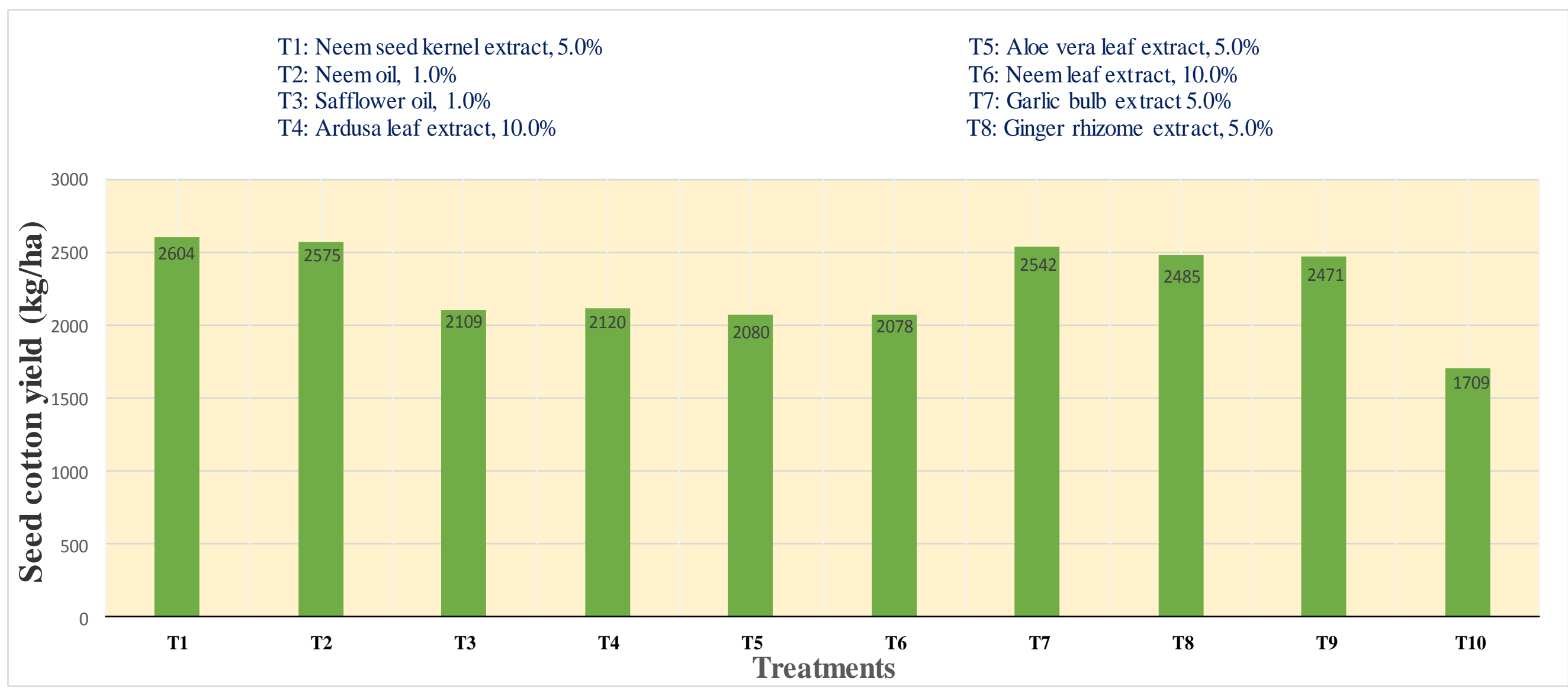




\section{Pooled over sprays}

Pooled over sprays results (Table 3 and Fig. 1) showed thatNSKE (3.46/leaf) was found significantly superior than all the evaluated botanicals except neem oil (3.54/leaf), NLE (3.66/leaf), GBE (3.78/leaf) and GRE (3.91/leaf). The plots treated with AVLE recorded the maximum (7.01/leaf) $S$. dorsalis population and it was at par with ALE (6.58/leaf), safflower oil (6.74/leaf) and TLE (6.84/leaf). From the above results, it can be deduced that the NSKE, neem oil, NLE, GBE and GRE were found more effective in reducing thrips population during present investigation.

\section{Effect on seed cotton yield}

The data on seed cotton yield were recorded in various botanical treatments as well as in control during study and are presented in Table 4 and Figure 2.

Maximum (2304kg/ha) seed cotton yield was recorded in plots treated with NSKE and it was at par with neem oil $(2295 \mathrm{~kg} / \mathrm{ha})$, NLE $(2275 \mathrm{~kg} / \mathrm{ha})$, GBE $(2252 \mathrm{~kg} / \mathrm{ha})$ and GRE $(2238 \mathrm{~kg} / \mathrm{ha})$. These five botanicals found relatively more effective which reflected on yield of seed cotton. The lowest $(1845 \mathrm{~kg} / \mathrm{ha})$ yield of seed cotton was recorded in plots treated with AVLE and it was at par with ALE (1902 kg/ha), safflower oil (1879 kg/ha) and TLE (1860 kg/ha).

Increase in yield over control in cotton crop was worked out for different botanical treatments and indicated that maximum $(49.71 \%)$ increase in yield found in plots treated with NSKE followed by neem oil (49.12\%), NLE (47.82\%), GBE (46.33\%) and GRE (45.42\%). Among the tested botanicals, minimum (19.88\%) increase in yield found in plots treated with AVLE followed by TLE (20.86\%), safflower oil (22.09\%)and ALE $(23.59 \%)$.

\section{Economics}

Looking to the NICBR, the highest (1: 7.34) return obtained with the treatment of NLE followed by NSKE (1: 6.20) and GBE (1: 4.54).The NICBR calculated 1: 2.85, 1: 2.65 and 1: 2.43 with treatments of neem oil, AVLE and GRE, respectively. The poor NICBR (1: 0.15 to $1: 0.80$ ) was recorded with the treatments of TLE, ALE and safflower oil.

Khattak et al., (2006) reported that neem oil 2 per cent and neem seed water extract 3 per cent significantly reduced the population of thrips, whiteflies and jassids on cotton up to 7 days after spray.Patel et al. (2009) evaluated some botanicals for the control of chilli thrips and revealed that the botanical formulations azadirachtin $(0.00075 \%)$ and neem seed kernel suspension $(5 \%)$ were found to be effective and gave more than $40 \%$ mortality of $S$. dorsalis. According to Khan et al., (2013), neem oil 2 per cent is proved to be most effective bringing about significant reduction in the population of thrips, jassids and whiteflies in $B t$ cotton and also garlic bulb and eucalyptus leaves extract gave significant result. Above reports drawn by various researchers are strongly supported the present findings.

In conclusion, among the nine botanicals evaluated against $S$. dorsalis infesting $B t$ cotton, application of neem seed kernel extract 5 per cent found the most effective followed by neem oil 1 per cent, neem leaf extract 10 per cent, garlic bulb extract 5 per cent and ginger rhizome extract 5 per cent with seed cotton yield 2304, 2295, 2275, 2252 and 2238 $\mathrm{kg} / \mathrm{ha}$, respectively. Looking to the NICBR, the highest (1: 7.34) return obtained with the treatment of neem leaf extract followed by NSKE (1: 6.20) and garlic bulb extract (1: 4.54). 


\section{References}

Abbas, M. A. (2004). M. A. General Agriculture. Publ. Emporium, 2nd (Ed.), Pakistan.

Anonymous (2017). ICAR-AICRP (Cotton) Annual Report (2016-17). ICAR-All India Co-ordinated Research Project (Cotton), Coimbatore-641 003, Tamil Nadu, pp. A, 2-5.

Capinera, J. L. (2001). Handbook of vegetable pests. Academic press, USA.

Khan, M. H., Ahmad, N., Rashdi, S. M. M., Rauf, I., Ismail, M. and Tofique, M. (2013). Management of sucking complex in $B t$ cotton through the application of different plant products. Pakhtunkhwa Journal of Life Science, 1(1), 42-48.
Khattak, M. K., Rashid, M., Hussain, A. S. and Islam, T. (2006). Comparative effect of neem (Azardirachta indica A. Juss) oil, neem seed water extact and baythroid TM against whitefly, jassid and thrips on cotton. Pakistan Entomology, 20 (1), 31-37.

Patel, B. H., Koshiya, D. J., Korat, D. M. and Vaishnav, P. R. (2009). Evaluation of some insecticides against chilli thrips, S. dorsalis Hood. Karnataka Journal of Agriculture Science, 22 (2), 327330.

Steel, R. G. D. and Torrie, J. H. (1980). Principles and procedures of statistics. Publ. McGraw Hill Book Company, New York, pp. 137.

\section{How to cite this article:}

Padaliya, S.R., R.K. Thumar, G.K. Pipaliya and Bhagora, J.K. 2018. Bio-efficacy of Different Botanicals against Thrips, Scirtothrips dorsalis Hood Infesting Bt Cotton. Int.J.Curr.Microbiol.App.Sci. 7(07): 2893-2903. doi: https://doi.org/10.20546/ijcmas.2018.707.339 